\title{
PLANETARY CAMERA OBSERVATIONS OF THE M87 STELLAR CUSP ${ }^{1}$
}

TOD R. LAUER

National Optical Astronomy Observatories, ${ }^{2}$ P.O. Box 26732, Tucson, Arizona 85726

\section{S. M. FABER}

UCO/Lick Observatories, Board of Studies in Astronomy and Astrophysics, University of California, Santa Cruz, Santa Cruz, California 95064

\section{ROGER LYNDS}

National Optical Astronomy Observatories, P.O. Box 26732, Tucson, Arizona 85726

WiLliam A. BAUM

Department of Astronomy, FM-20, University of Washington, Seattle, Washington 98195

\section{S. P. EWALD}

Space Telescope Science Institute, 3700 San Martin Drive, Baltimore, Maryland 21218

EDWARD J. GRoth

Department of Physics, Jadwin Hall, Princeton University, Princeton, New Jersey 08544

J. JEFF HESTER

Infrared Processing and Analysis Center, 100-22, California Institute of Technology, Pasadena, California 91125

Jon A. HoltzMAN

Lowell Observatory, 1400 Mars Hill Road, Flagstaff, Arizona 86001

JEROME KRISTIAN

The Observatories, Carnegie Institution of Washington, 813 Santa Barbara Street, Pasadena, California 91101

ROBERT M. LIGHT

UCO/Lick Observatories, Board of Studies in Astronomy and Astrophysics, University of California, Santa Cruz, Santa Cruz, California 95064

EARL J. O'NeIL, JR.

National Optical Astronomy Observatories, P.O. Box 26732, Tucson, Arizona 85726

DONALD P. SCHNEIDER

Institute for Advanced Study, Princetion, New Jersey 08540

EDWARD J. ShaYa

Department of Physics and Astronomy, University of Maryland, College Park, Maryland 20742

JAMES A. WESTPHAL

Division of Geological and Planetary Sciences, 170-25, California Institute of Technology, Pasadena, California 91125 Received 2 October 1991; revised 12 November 1991

\section{ABSTRACT}

Analysis of $V$ and $I$ band $H S T$ Planetary Camera images of the giant elliptical galaxy M87 show that its central starlight distribution is consistent with the black hole $\mathscr{M}_{\bullet}=2.6 \times 10^{9} \mathscr{M}_{\odot}$ cusp model proposed for M87 by Young et al. [ApJ, 221, 721 (1978)]. A combined approach of image deconvolution and modeling is used to investigate the starlight distribution into limiting radii of $\approx 0$ ".04 ( $3 \mathrm{pc}$ at 16 $\mathrm{Mpc}$ ). The central structure of M87 can be described by three components: a power-law starlight profile of the form $\mu(r) \propto r^{-1 / 4}$ for $r<3^{\prime \prime}$, a central nonthermal point source, and optical counterparts of the jet knots N1 and M identified by VLBI observations. M87 lacks a constant surface brightness core, and its central starlight luminosity density exceeds $10^{3} L_{\odot} \mathrm{pc}^{-3}$ ( $I$ band) for $r<10 \mathrm{pc}$. The profile strongly resembles a stellar cusp associated with a massive black hole. A review of existing velocity dispersion observations suggests that the Young et al. black hole mass can be accommodated to the observations with minor adjustments of dynamical models. The central luminosity spike itself remains unresolved at $H S T$ resolution, with $r_{\mathrm{c}}<1 \mathrm{pc}$. The spike has optical spectral index $\alpha=-0.46 \pm 0.20$ and is at least as blue if not bluer than the rest of the M87 jet. The total nonthermal flux in the inner 1" of M87 agrees well with the central radio flux and the radio-optical spectral index of the rest of the jet. It is also consistent with the spectral-line dilution seen by Dressler \& Richstone [ApJ, 348, 120 (1990)]; we thus argue that the spike is completely nonthermal.

\footnotetext{
${ }^{1}$ Based on observations with the NASA/ESA Hubble Space Telescope, obtained at the Space Telescope Science Institute, which is operated by AURA, Inc., under NASA Contract No. NAS 5-26555.

${ }^{2}$ The National Optical Astronomy Observatories are operated by the Association of Universities for Research in Astronomy, Inc. (AURA) under cooperative agreement with the National Science Foundation.
} 


\section{INTRODUCTION}

One of the primary missions of the Hubble Space Telescope is to look for evidence of massive black holes in galactic nuclei, and M87 is one of the prime targets. The first arguments that M87 might harbor a massive black hole were raised by Young et al. (1978) and Sargent et al. (1978). Young et al. (1978) argued for a black hole mass $\mathscr{M}_{\odot}=(2.6 \pm 0.5) \times 10^{9} \mathscr{M}_{\odot}$ based on the departure of the M87 brightness profile within $r<10^{\prime \prime}$ from an isothermal model. The Young et al. model includes a central starlight cusp that continues into the center as a power law, but assumes that the well-known central luminosity spike itself is a nonthermal point source that is not relevant to the stellar distribution. Sargent et al. (1978) argued for a mass $\mathscr{M}_{\bullet}=5 \times 10^{9} \mathscr{M}_{\odot}$ based on the central velocity dispersion profile, which they observed to rise to $\sigma \approx 340 \mathrm{~km} / \mathrm{s}$ at $r \approx 2^{\prime \prime}$. Both the Young et al. and Sargent et al. papers were published together and are usually linked in citations; however, as we will emphasize later, it is important to remember that the respective photometric and dynamical arguments were essentially independent and have been linked only by more recent analysis strategies.

Much subsequent work on the M87 nucleus has been directed toward testing the Young et al. and Sargent et al. conclusions. Dressler (1980) and Dressler \& Richstone (1990) measured velocity dispersions interior to the Sargent et al. resolution limit and found a $\sigma$ of only $360 \mathrm{~km} / \mathrm{s}$ within $r \approx 1.0 "$. While it is not clear whether the Dressler (1980) measurement was properly centered on the nucleus, the Dressler \& Richstone (1990) spectral observations show strong dilution by a central nonthermal source and thus appear to be well within the central seeing disk; the observed dispersion is much lower than the Sargent et al. prediction of $\sigma \sim 500 \mathrm{~km} / \mathrm{s}$ at that location. Similar conclusions were reached by Jarvis \& Peltier (1991). Jarvis \& Melnick (1991) observed $\sigma \approx 460 \mathrm{~km} / \mathrm{s}$ over the central 3" of M87 by use of the $\mathrm{Ca}$ II triplet, but this result conflicts with all previous dispersion measures over the same region.

Less work has been done to test the photometric analysis of Young et al. (1978), although Zavatti et al. (1984) and Bagnoulo \& Chambers (1987) have shown that the central spike remains unresolved at the subarcsecond level. While Young et al. argued that the spike was nonthermal, if at least part of its light comes from a compact central star cluster, this would have an important effect on interpreting the inner velocity dispersion profile. For $r>2$ ", Lauer (1985a) showed that the gently sloping nonisothermal brightness profile that Young et al. (1978) took as evidence for a black hole is not unique to M87 and is in fact common to most giant elliptical galaxies with well resolved cores. Kormendy (1985a) reached a similar conclusion. Both papers showed that "normal" isothermal cores are rarely if ever present in elliptical galaxies, bringing into question the Young et al. point of departure for modeling M87.

On the theoretical side, Duncan \& Wheeler (1980) explored the possible importance of nonisotropic velocity ellipsoids for modeling central dynamics, a point not considered by Sargent et al. (1978). More refined analyses by Binney \& Mamon (1982), Richstone \& Tremaine (1985), and Dressler \& Richstone (1990), to name a few, all emphasized the enormous freedom that models have to fit both the photometric and dispersion observations if the assumption of an isotropic velocity field is relaxed, even without a black hole. The general conclusion from work to date is that while M87 may harbor a central black hole of substantial mass, such an object is not required and is perhaps not even favored to explain either the light or the dispersion profile.

In fact, M87 is a difficult target for detection of a central black hole; the high dispersion of the background stellar population means that one must look quite close to even a massive hole to see its effects. A black hole dominates the region of the nucleus only within $r<140\left(\mathscr{M}_{\bullet} / 4 \times 10^{9} \mathscr{M}_{\odot}\right)(\sigma / 350 \mathrm{~km} / \mathrm{s})^{-2}$ pc (Young 1980 ), where $\sigma$ is the "background" stellar dispersion. For an adopted distance to M87 of $16 \mathrm{Mpc}$, which implies $1^{\prime \prime}=77.6 \mathrm{pc}$, these radii all lie within $2^{\prime \prime}$ and thus all groundbased observations for the black hole masses under consideration will be strongly affected by seeing.

A common theme expressed by many of the references quoted above is that $H S T$ observations might decisively demonstrate the existence of a central black hole. Even with the strong spherical aberration present in the HST primary (Burrows et al. 1991), it appears that $H S T$ can still obtain unique high resolution information on galactic nuclei (e.g., NGC 7457 Lauer et al. 1991). We present here an analysis of Planetary Camera images of M87 in the near infrared and visible. We show that the central stellar brightness distribution of M87 does not level off at the center, but continues into $r=0$ approximately as a power-law cusp of the form $\mu(r) \propto r^{-1 / 4}$, reaching central luminosity densities $\rho_{L}>10^{3} L_{\odot} \mathrm{pc}^{-3}$ within $r<10 \mathrm{pc}$. This simple picture is indistinguishable, given the errors, from the photometric model originally advanced by Young et al. (1978), but now tested at smaller radii.

\section{OBSERVATIONS AND ANALYSIS}

\subsection{Observations and Basic Reduction}

HST observations of M87 were obtained on day 152 of 1991 with the PC, or high resolution mode, of the Wide Field/Planetary Camera (WFPC). Detailed description of the camera is presented in Griffiths (1989). Briefly, the PC comprises four $\mathrm{CCD}$ cameras imaging a contiguous $66^{\prime \prime} \times 66^{\prime \prime}$ field of view. Each CCD camera has an $800 \times 800$ pixel format with 0 "044 per pixel. Two $700 \mathrm{~s}$ exposures were obtained with filter F785LP, which corresponds roughly to the Johnson $I$ band; the observations were done in this color to minimize the contribution of the blue spike with respect to the underlying starlight. Observations in the $V$ band ( F555W) were obtained earlier in the year, but with poor PSF data and positioning for study of the nucleus. These were used only to estimate the color of the central spike (see below).

The maximum signal level in each $I$ image was $1.5 \times 10^{4} e^{-}$per pixel in the spike. The telescope was guided in coarse track during the exposure sequence. The guiding jitter was only $0.014 \mathrm{rms}$, which is excellent performance for coarse track. There are no obvious effects of this small amount of jitter in either image, and the signal levels in the nucleus agree well between the two images. The nucleus was positioned close to the center of CCD PC6, 12" away from the nearest edge of the PC6 field; the positions of the nucleus in the two exposures agree at the milliarcsecond level. Two hours after the M87 exposures, two $0.6 \mathrm{~s}$ and one $1.2 \mathrm{~s}$ exposures were taken of a nearby SAO star to characterize the PSF. The star was positioned 1".4 from the CCD location of the nucleus; the slight offset was due to a small pointing 
error. The exposure level in the $1.2 \mathrm{~s}$ exposure was $2.6 \times 10^{4} e^{-}$in the central pixel.

At the time of the observations, calibration of the WFPC was still in a preliminary state; however, all reduction steps outlined by Lauer (1989) could be completed. The flatfield for F785LP was generated from several earth "streak" frames and gave cosmetically excellent results. Cosmic ray events were rejected by intercomparison of the two galaxy pictures; the analysis described below was performed on the sum of the two exposures cleaned of cosmic rays. The two short PSF exposures were used to reject cosmic rays in the $1.2 \mathrm{~s}$ exposure, but as the faint halo of the PSF was dominated by readout noise in the short exposures, only the $1.2 \mathrm{~s}$ exposure was used to represent the PSF.

The reduced $I$ image of M87 is presented in Fig. 1 [Plate 53] (only the PC6 portion is shown). The stellar nature of the central luminosity spike is clearly evident. The positioning allowed almost all of the jet to be observed, which will be the subject of a future paper. Several globular clusters are visible as pointlike sources scattered about the core. The depth of the present images for globulars is slightly shallower than the CFHT images used by Lauer \& Kormendy (1986). Further analysis of the globular clusters will not be presented here, although object identification from the present images agrees well with the object list of Lauer \& Kormendy (1986).

The deconvolved $I$ image after 80 Richardson-Lucy iterations (Richardson 1972; Lucy 1974) is presented in Fig. 2 [Plate 54]. This is shown to provide a qualitative impression of what M87 looks like corrected for spherical aberration although this image is not used for the analysis described below. Strongly evident is the central gradient in the galaxy light distribution that continues into the nucleus.

\subsection{Strategy for Measurement of the M87 Starlight Profile}

The strength of the central luminosity spike in M87 plus the strong spherical aberration smearing makes measurement of the central light distribution of M87 challenging. Accurate observation of the PSF is crucial. Fortunately the PSF is critically sampled in the passband of the observations. We can thus shift the PSF and resample the observations by sinc interpolation, which is the preferred interpolation method for properly sampled data (Bracewell 1978). The analysis will be done in two stages. In the first stage we start with rough subtraction of the luminosity spike, followed by Richardson-Lucy deconvolution of the remaining light to obtain an initial model of the central starlight profile; this leads to further isolation of the spike and jet components, improving knowledge of the light profile at smaller radii. In the second stage, we fit PSF-convolved multicomponent models to the image directly to provide the final description of the central starlight profile; thus while we use deconvolution to guide our intuition and modeling, in the end we do not depend on it for details of the light distribution in the innermost regions. The final model of the central light distribution of M87 that we derive is simple, consisting of an essentially pointlike central spike, a small contribution from two inner jet components, and a shallow power law for the underlying starlight.

\subsection{Initial Spike Subtraction and Starlight Model}

The raw profile of M87 measured directly from the $I \mathrm{im}$ age with the high-resolution ellipse fitting software of Lauer

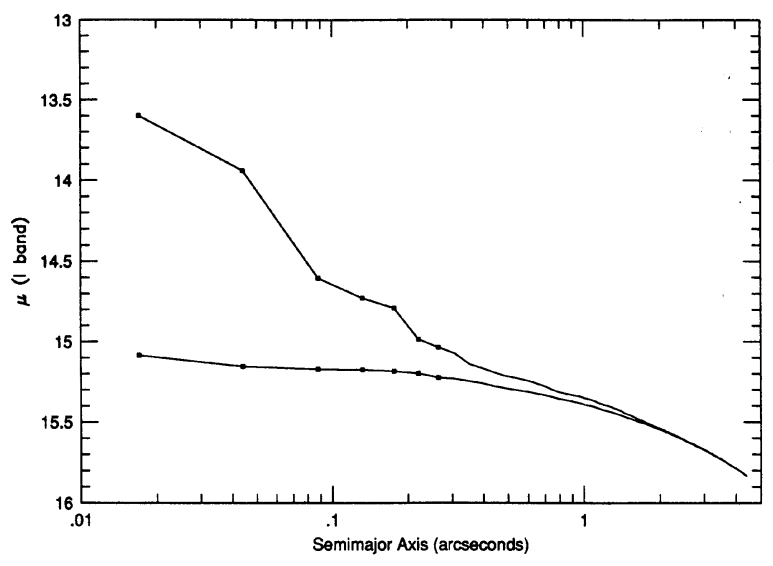

FIG. 3. I band surface brightness profiles of M87 prior to deconvolution before and after subtraction of the luminosity spike and inner jet (individual points in the profile are only shown for $r<0$."3). The final spike and jet model discussed in Sec. 2.4 has been used for the subtraction. Note the small contribution of the nonthermal sources to the brightness profile for $r>0$ ". 3 .

( $1985 b)$ is presented as the top curve in Fig. 3. The contribution of the nuclear spike is strong at the center, but for $r>0$." 1 , the underlying starlight dominates the profile; thus even crude subtraction of the spike allows accurate measurement of the starlight profile at subarcsecond radii. The initial subtraction of the spike was done just by shifting the PSF star to the nucleus location and scaling it so that the central pixel in the difference image was no brighter than its immediate neighbors. To first order the spike is a point source, so this procedure worked well. In fact, the spike is sharp enough so that its first diffraction ring is clearly visible in the M87 image and is seen as the bump in the luminosity profile at $r \approx 0 " .2$.

For $r>0$ " 4 , the contribution of the spike to the local surface brightness is less than $0.1 \mathrm{mag}$ and vanishes by $r \approx 2^{\prime \prime}$. Over the same region, the starlight profile shows a substantial gradient that is much larger than any uncertainties (although the lower curve in Fig. 3 is the light left behind after the more sophisticated subtraction of central nonthermal light described below). Richardson-Lucy deconvolution of this first "cleaned" image of the galaxy center suggested that the galaxy light profile might follow a power law for $r<1$ "2. In fact, a fit to the deconvolved profile for 0 ". $6<r<1$ ". 2 showed that the brightness profile over this region can be described by a power law with slope $\gamma=-0.26 \pm 0.01$ with rms residuals of $0.01 \mathrm{mag}$.

The next step was to see if this power law could be extrapolated into $r<0$ " 6 . The isophotes are nearly circular over this region and were assumed to be circular for the inward extrapolation. Figures 4(a) and 4(b) [Plate 55] show this model convolved with the PSF and subtracted from the observations; the spike is now neatly isolated. Subtraction of a scaled point source from the residuals reveals a small portion of the jet emerging from the nucleus to the upper right and a small residual component of the spike itself offset by a fraction of a pixel in the opposite direction. Otherwise there is no evidence of systematic positive or negative residuals that would indicate that the power law model has either under or overestimated the central starlight contribution [Fig. 4(c)]. 


\subsection{Refinement of the Spike and Central Jet Contributions}

We now take a closer look at the spike and jet to account for the residual structure seen near the central point source. Describing this structure presented some difficulties as neither the jet components nor the spike residuals appeared to have a form that could be simply modeled as a point source. Direct Richardson-Lucy deconvolution of the residuals was instructive but ultimately not useful because the finite resolution of the deconvolved image made it difficult to understand the true spatial extent of the nuclear features. Deconvolution replaces the original PSF with one that is sharper but that still has a significant width-in the direct analysis of a deconvolved image we still must be content with the residual blur of the deconvolved PSF. The approach taken was to start over and model the complete spike plus jet (after subtraction of the stellar power law) as a set of point sources by use of a CLEAN algorithm (Högbom 1974); in this way, we test the spatial extent of the residuals directly. This was done on a grid twice as fine as the original observations to allow approximately for displacement of components from the original pixel centers.

It was found that all of the nuclear structure could be described by three clusters of CLEAN components, with one set corresponding to the spike, and the other two to separate knots in the jet. The CLEAN analysis confirmed that the spike could be described as a nearly pure point source. $81 \%$ of the light in the spike cluster of components was in the single pixel at the position of the spike centroid; the remaining $19 \%$ of the light was in components offset opposite to the jet but still within 0.022 (a single subpixel) of the main spike. The total luminosity in the offset spike components is consistent with the recorded rms guiding jitter, though perhaps not with its detailed distribution. The two M87 exposures that were combined for the present analysis both show similar structure, indicating that an offset spike component could be a real feature of the images. However, because of the jitter we are not confident of its physical reality and will assume for the discussion below that it is just part of the central spike. By experimenting with small amounts of broadening in an attempt to account for all components associated with the spike, we limit any intrinsic width of the central spike to $<0$ ".02 FWHM or core radius $r_{\mathrm{c}}<1 \mathrm{pc}$. This result is not surprising if the spike is purely nonthermal, since VLBI observations show the radio spike to have FWHM $<2 \times 10^{-4}$ arcsec (Spencer \& Junor 1986); it is interesting in the optical context, however, as it shows that any central star cluster must be either exceedingly compact or faint (see below).

We subtract this refined model of the spike to get a better view of the inner jet. Figure 4(d) shows the jet residuals with the spike subtracted. In the the light that remains, the CLEAN analysis identifies two clusters of components spaced 0"09 and 0 . 18 from the spike. These appear to correspond almost exactly to the components N1 and M identified in the VLBI observations of Reid et al. (1989). Oddly, we see no evidence for component N2 that Reid et al. (1989) predict should be separated from the spike along the same direction by 0.029 at the time of the present observations. While it is possible that this light is too close to the spike to be separated from it, we emphasize that the subtle extension of the spike that we discussed above is similar to that expected for N2, but in the opposite direction. Reid et al. (1989) show that the position of N2 slowly varies with time; without current VLBI observations, we cannot tell whether in fact our small counter feature is related to $\mathrm{N} 2$.

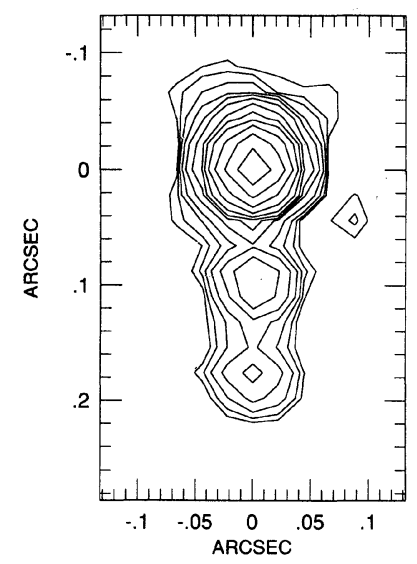

Fig. 5. Contour map of the deconvolved luminosity spike and inner jet. This figure shows the CLEAN components (see text) restored with a one pixel (0.044 FWHM) Gaussian beam. The jet has been rotated to be parallel with the figure axes. The contours are in halfmagnitude steps, but are otherwise arbitrary. Jet knot N1 is the knot closest to the spike; knot $\mathrm{M}$ is the knot 0 " 18 distant from the spike.

The final image residuals after subtraction of the jet, spike, and power law are shown in Fig. 4(e). The residuals are flat with a mean of zero and a dispersion nearly consistent with photon noise. The final adopted structure for the spike and inner jet is shown as a contour plot in Fig. 5, which shows the CLEAN components restored with a 0.044 FWHM Gaussian beam. The final starlight profile measured after subtraction of the spike and jet is shown as the lower line in Fig. 3; the center of the galaxy after subtraction of the spike and jet models (but not the starlight model) is shown in Fig. 4(f).

\subsection{The Central Luminosity Spike}

The isolation of the spike that is possible with the present observations allows us to resolve the longstanding controversy on whether the spike is a star cluster or a nonthermal source like the jet. Young et al. derive a spike $m_{V}=16.69 \pm 0.05$, with $B-V=0.73$. Biretta et al. (1991) find $g-r=0.32$, which implies $B-V=0.86$. These colors are $0.2-0.3$ mag redder than the innermost jet knots (Perez-Fournon et al. 1988; Biretta et al. 1991) and thus suggest that some of the light may be starlight. However, colors from the ground are very sensitive to both the assumed galaxy profile and the seeing correction so this small difference may not be significant.

An alternative approach is to compare dilution of the stellar line strengths with the amount of light expected from the spike. Dressler (1980) claimed at most only a $20 \%$ dilution through a central $1^{\prime \prime} \times 1^{\prime \prime}$ aperture, which was far smaller than the Young et al. photometry would predict for a pure synchrotron source. Dressler therefore concluded that a large fraction of the spike light must be stellar. However, with newer data and more careful technique, Dressler \& Richstone (1990) found the line-strength dilution to be $35 \%-40 \%$ within the central $0.50 \times 0$ ". 72 aperture in 1 ". 15 seeing (FWHM). Comparing this to the observed light profile along the slit, they concluded that this dilution was consistent with pure synchrotron emission.

Observing in the red at $8500 \AA$ (which is close to the 8900 $\AA$ effective wavelength of F785LP), Jarvis \& Melnick (1991) claimed that the Ca II triplet absorption line strength remained constant within the central 1" of M87. To reconcile this with the dilution seen at bluer wavelengths, these authors postulated a very young blue star cluster whose red 
continuum was produced by cool supergiants with strong Ca II absorption. They also claimed to resolve the central spike, for which they gave a core radius (HWHM) of 1."15. This value, plus their central dispersion of $450 \mathrm{~km} / \mathrm{s}$, indicated a cluster mass of $3.5 \times 10^{9} \mathscr{M}_{\odot}$ and a mass-to-light ratio $\mathscr{M} / L_{V} \approx 60$. As this is much higher than expected for a young stellar population, they were led to conclude that the nucleus of M87 contains large amounts of dark matter.

We can improve on these arguments in three ways. To start with, we can rule out the Jarvis and Melnick picture of a central star cluster that contains a significant fraction of the M87 core mass, $\mathscr{M}_{\mathrm{c}} \sim 4 \times 10^{9} \mathscr{M}_{\odot}$. As noted above, we find the spike to be unresolved at the $1 \mathrm{pc}$ level; any core present is at least 90 times smaller than the Jarvis \& Melnick (1991) result. Even if we assume that the spike is a star cluster with central $\sigma=450 \mathrm{~km} / \mathrm{s}$, this new radius reduces the mass to only $5 \times 10^{7} \mathscr{M}_{\odot}$, which implies $\mathscr{M} / L_{V} \approx 1$, given the spike luminosity quoted below.

Second, the HST data provide more reliable colors and fluxes for the spike, clarifying the relationship of the spike to the central radio source and the rest of the optical jet. We derive F785LP magnitudes of 16.20 for the spike, and 18.89 and 19.78 for the jet components $\mathrm{N} 1$ and $\mathrm{M}$; these correspond to fluxes of $0.81,0.068$, and $0.030 \mathrm{mJy}$, respectively, using the $I$ flux calibration of Johnson (1966). At groundbased resolution, all components added together give total $m_{I}=16.08$, or $0.90 \mathrm{mJy}$. The poorer PSF of the F555W images allows determination of only the total $V$ magnitude for the spike plus inner jet. For this we find $m_{V}=16.81$, or $0.72 \mathrm{mJy}$; this gives $V-I=0.73$, for the sum of all inner components, which is a full magnitude bluer than the $V-I=1.73$ for the underlying starlight. The implied spectral index is $\alpha=-0.46 \pm 0.20$, where the error reflects a probable uncertainty of $\pm 0.1 \mathrm{mag}$ in the color zero point. Previous optical work on the other knots in the M87 jet has not yielded consistent optical spectral indices, although if anything they seem redder than our colors for the inner components. Keel (1988) finds $\alpha \sim-0.7$ for the knots of the jet within 10" of the nucleus, while Biretta et al. (1991) found $\alpha \sim-1.2$ over the same region. On balance, the blueness of the central components relative to both the starlight and the jet strongly supports a purely nonthermal origin for the central spike. This view is also supported by the radio-optical indices. Comparison of the present spike fluxes to the radio fluxes given by Biretta et al. gives a radio $F 785 \mathrm{LP}$ $\alpha=-0.74$, which compares well with the radio-optical $\alpha=-0.64$ that Biretta et al. find for the jet outside the spike. Taken together, the data indicate strongly that the spike is nonthermal and that its optical properties agree, within the errors, with those expected from the radio morphology of the M87 jet.

Last, we find that our description of the M87 core agrees well with the spectral line dilution estimated by Dressler \& Richstone (1990). We took the $I$ model for the central spike plus jet and rescaled it to $V$, assuming the relative (galaxyspike) color noted above. To this we added the model of the starlight, under the assumption that the stars have constant $V-I$ color with radius. We then convolved this combination with a 1".15 FWHM Gaussian seeing profile and sampled the result with the Dressler and Richstone 0 " $50 \times 0$ ". 72 aperture centered on the spike. The resultant dilution factor was measured as $42 \%$, completely consistent with the Dressler and Richstone estimate quoted above, again suggesting that the spike is essentially all synchrotron radiation.

\subsection{Limits on the Central Starlight Profile}

At this point we have shown that the central 1" of M87 can be modeled as a pure $\gamma=-0.26$ power law for the starlight, a central, virtually unresolved point source, and two jet components. The problem now is to establish the significance and uniqueness of this model and, in particular, the errors on the starlight component. The first question that we will address is the one that we consider scientifically most interesting, namely whether we are really seeing a power law that continues into the very center or whether the starlight profile might instead slowly flatten. To test this hypothesis, we fitted the image with two-dimensional surface brightness distributions of the form

$$
\mu(r)=\left(a^{2}+r^{2}\right)^{-0.13}
$$

plus the CLEAN reconstruction of the central spike (the two jet components were subtracted ahead of time and were not fitted). The amplitudes of the starlight and central spike were free to vary separately. At $r=a$ the local logarithmic slope of the starlight distribution is $\gamma / 2$ and goes to zero at $r=0$. Models were generated for $a=0.00,0$. 05,0 ". 10,0 ".20, and 0 " 40 . As $a$ increased, the models became flatter and contributed less light to the galaxy center. The strength of the spike increased to compensate, but for the models with $a>0$."05, this always led to a poor fit-typically the model spike would be too bright at $r=0$, but the spike plus model would fall below the observations at slightly larger radii, showing that an extended starlight component on top of that provided by the model was required. The best fit was obtained with the $a=0$ ".00 model, although the $a=0$ ".05 model was only slightly poorer. If $a$ was allowed to vary as a free parameter, the fit always produced $a<0$."05. The increase in the central spike luminosity in going from $a=0$ ". 00 to 0 " 10 is only $3 \%$.

The final brightness profile after subtraction of the spike and jet shown in Fig. 3 is presented again in Fig. 6 along with these models; the $a=0$ ". 00 model fits the data for all $r<0$ ". 6 to within a few hundredths of a magnitude. As another measure of the true distribution of starlight, we also show the final profile after 80 iterations of Richardson-Lucy deconvolution, as well as the $a=0$ ".00 model prior to convolution. The centralmost point in the deconvolved profile falls below the model by $0.3 \mathrm{mag}$; however, some of this shortfall is doubtless due to the limited power of deconvolution, which can boost the resolution by only so much. The two profiles agree to $0.1 \mathrm{mag}$ everywhere else, and the differences merely reflect amplification of the small residuals noted above prior to deconvolution. For convenience, we give both the deconvolved profile and the best fitting model with $\gamma=-0.26$ in Table 1.

From comparing the $\gamma=-0.26$ models in both observed (Fig. 3) and deconvolved (Fig. 6) space, one sees a slight excess of light in the galaxy near $r \sim 0$ compared to the model. This can be largely removed by steepening $\gamma$ in the model to -0.30 . In this case, the limit on $a$ increases somewhat to 0 ".05, but is still small. This example gives some feeling for the range of $\gamma$ and $a$ allowed in the power-law models. Values of $\gamma$ much steeper than -0.30 are excluded, as they exceed the light actually seen near $r \approx 0$ ". 3 , where the deconvolution is quite stable and reliable. In short, we conclude that $\gamma=0.26 \pm 0.04$ for $0.1<r<0$ ". 6 .

It is difficult to place formal uncertainties on the intrinsic 


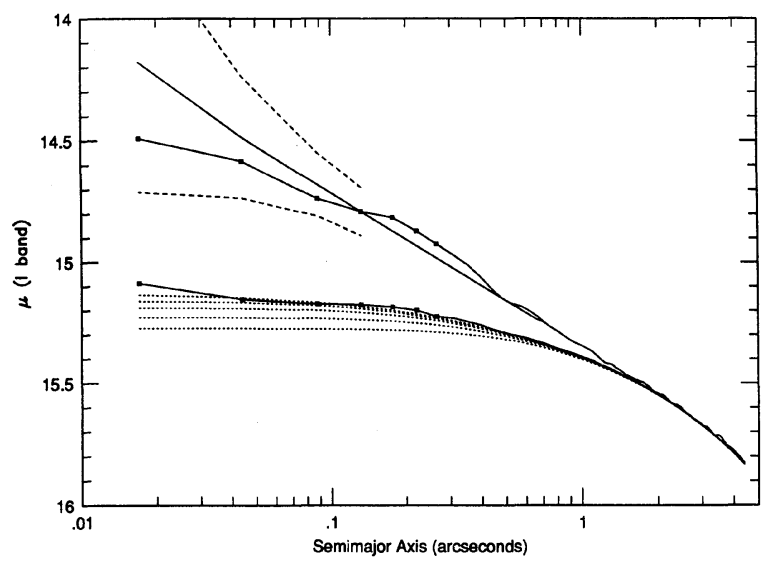

FIG. 6. Starlight profiles of M87 before and after deconvolution. The lower solid line is the starlight profile prior to deconvolution already shown in Fig. 3 measured after subtraction of the spike and inner jet. The two upper solid lines show this profile after 80 Richardson-Lucy deconvolution iterations and the pure power-law model with $\gamma=-0.26$ and $a=0.00$ prior to PSF convolution. The bracketing dashed lines are an "error envelope" for the deconvolved profile (see text). The lower set of dotted lines are the various models after PSF convolution matched to the observed starlight profile. All models assume $\gamma=-0.26$. The uppermost has $a=0$ ". 00 ; note that this model matches the observed starlight profile to a few hundredths of a magnitude at all radii. The next lower model shown has $a=0$."05; $a$ increases downward in powers of two until $a=0$ ". 40 .
TABLE 1. M87 surface brightness profiles.

\begin{tabular}{|c|c|c|c|c|c|c|c|c|}
\hline$R$ & $\mu_{I}$ & P.A. & $\epsilon$ & Model & $R$ & $\mu_{I}$ & P.A. & $\epsilon$ \\
\hline 0.017 & 14.491 & 3.6 & 0.160 & 4.179 & 1.672 & 15.489 & 53.8 & 0.040 \\
\hline & & & & & & & & \\
\hline 0.088 & 14.73 & 163.6 & 0.112 & & 1.760 & 15.497 & 60.3 & 0.072 \\
\hline 132 & 14.790 & 163.6 & 0.000 & 14.790 & 1.804 & 15.507 & 53.8 & 0.072 \\
\hline 0.176 & 14.814 & & 0.147 & 14.870 & & 15.514 & 53.8 & 0.072 \\
\hline 0.220 & 14.87 & 146.7 & 0.147 & 14.932 & 1.892 & & 62.5 & 0.007 \\
\hline 0.264 & 14.925 & 136.6 & 0.162 & & 1.936 & & 62.5 & 0.005 \\
\hline 0.308 & 14.971 & 125.5 & 0.162 & 15.026 & 1.980 & 15.539 & 111.1 & 0.005 \\
\hline 0.352 & 15.013 & 119.0 & 0.162 & 15.063 & 2.024 & 15.544 & 105.1 & 0.005 \\
\hline 0.39 & 15.06 & 118.4 & 0.138 & 15.0 & 2.068 & & 100.3 & 0.015 \\
\hline 0.440 & 15.114 & 118.4 & 0.131 & 15.125 & 2.112 & 15.5 & 100.3 & 0.015 \\
\hline 0.484 & 15.152 & 114.0 & 0.118 & .151 & & 15.5 & 99.3 & 0.015 \\
\hline 0.52 & 15.17 & 107.9 & & 5 & & & & \\
\hline 0.57 & 15.183 & 114.0 & 0.119 & 5.198 & 2.244 & 15.5 & 90.5 & 0.018 \\
\hline 0.6 & 15.2 & 118 & 0. & & & 15. & & 0.024 \\
\hline & 15.2 & 118 & 0.0 & 15.2 & & & & 0.034 \\
\hline 0.70 & 15.248 & 119.5 & 0.079 & - & 2.376 & 15.5 & 83 & 0.060 \\
\hline 0.74 & 15.2 & 133 & 0.09 & & 2.4 & 15.5 & & 0.060 \\
\hline & 15.2 & 147 & & & & & & 0.060 \\
\hline & 15.30 & 147.4 & & - & & & & \\
\hline 0. & 15.317 & 147.8 & 0.08 & - & 2.5 & 15.616 & 96.8 & 0.037 \\
\hline 0. & 15.3 & 147 & & & & & & 0.012 \\
\hline & & 147 & & - & & & & \\
\hline 1.01 & 15.34 & 146.5 & 0.06 & - & 2.6 & 15.632 & 133.0 & 0.003 \\
\hline 1.0 & & & & & & & & 0.003 \\
\hline & & & & - & & 15. & & \\
\hline 1. & 15.3 & & 0.0 & - & 3.270 & 15.7 & 59. & 0.005 \\
\hline 1 & 15.4 & & & & & & & 0.012 \\
\hline 1. & 15.4 & & & - & & & & \\
\hline 1. & 15.42 & & & - & & 15. & & 0.017 \\
\hline 1. & & & & & & & & 0.020 \\
\hline & 15.4 & & 0.0 & - & 7.3 & 16. & 8.1 & 0.031 \\
\hline & & & & - & & & & 0.03 \\
\hline & & & & & & & & \\
\hline 1.496 & 15.46 & 67 & 0.0 & - & 12.0 & 16.6 & 1. & 0.038 \\
\hline & 15.4 & & & & & 16.8 & 175 & 0.034 \\
\hline 1.58 & 15.4 & 67 & & - & & & & 0.029 \\
\hline 1.628 & 15.484 & 53.8 & 0.038 & & 19.530 & 17.343 & 165.3 & 0.031 \\
\hline
\end{tabular}

It might be argued that all of these fitting exercises have merely returned the model assumptions that were used to isolate the nonthermal spike; however, since the spike is essentially pointlike, its contribution to the profile between 0 ". 1 and 0.6 is well constrained and well determined by the light

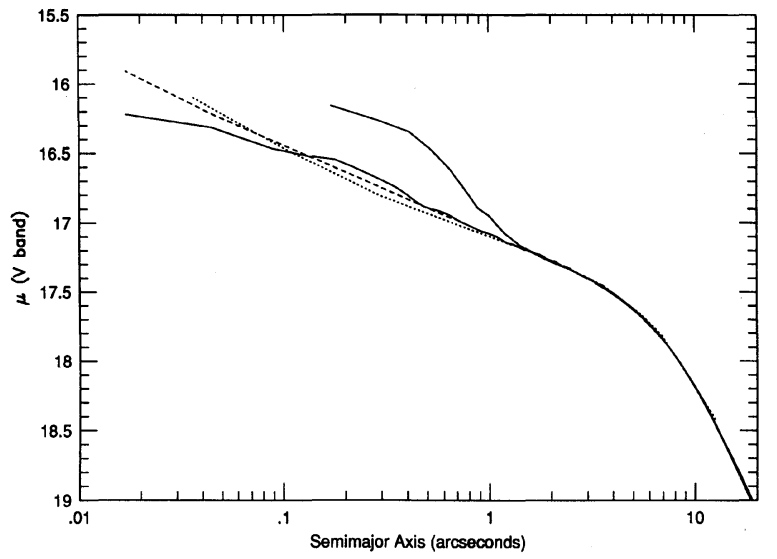

FIG. 7. The present $I$ band starlight profile normalized to the Young et al. (1978) ground-based $V$ band observations and black hole cusp model. The solid lines show the Young et al. $V$ profile and the deconvolved $I$ band profile. The $\gamma=-0.26$ power-law model is shown as a dashed line. The Young et al. cusp model is shown as a dotted line. 
within $r=0$ " 05 . We have merely assumed that all pointlike light components are nonthermal and that the starlight is comprised of everything but the pointlike components. Under this assumption the separation is quite clean. In passing, we note that we have assumed that the center of the galaxy is coincident with the luminosity spike; after the removal of the nonthermal components as described, we find no evidence that the starlight has a different center to limits of 0.015 or 1 pc.

Last, as a final check, one of us (CRL) measured the starlight profile independently of the analysis presented above. For the check analysis, the PSF was represented by a weighted sum of all three PSF exposures, rather than just the brightest exposure alone, and third-order Stirling interpolation was used to shift the PSF to the spike location rather than sinc interpolation. The amplitude of the luminosity spike was derived by a bright-end extrapolation of the covariance between individual PSF and galaxy pixels-no underlying model of the starlight was assumed. The residual jet was removed by interactively subtracting point sources until it could no longer be seen. The final starlight profile prior to deconvolution thus derived agrees with the present one to $0.01 \mathrm{mag}$ at $r=0$ and for $r \geqslant 0$ " 1 , and is 0.10 mag brighter than the present profile for 0 " $04<r<0$ " 10 . This verifies the conclusion above that the deconvolved profile is well determined for $r \geqslant 0$. 1, and if anything may be brighter than our present results for $r<0$ ". 1 .

\section{DISCUSSION}

The M87 brightness profile that we present here is a striking departure from what conventional models for a "normal" giant elliptical core would be expected to look like at $H S T$ resolution. M87 does not possess a flat constant surface brightness core. From Fig. 6, any core present must have $r_{\mathrm{c}}<0$ ". 5 , where $r_{\mathrm{c}}$ is defined as the point at which the brightness profile falls to half of its central intensity. The light distribution consists of a steep outer power-law, $\mu \sim r^{-1.3}$, beyond $r>10^{\prime \prime}$, smoothly changing over to a shallow $\mu \sim r-0.26$ power law within $r<3^{\prime \prime}$. The radius of maximum logarithmic curvature occurs at $r_{2}=7.0=540 \mathrm{pc}$, which is slightly less than the value of the Young et al. core radius $\left(r_{\mathrm{c}}=9.6=745 \mathrm{pc}\right)$. These parameters are impressively close to the black hole cusp model fitted to M87 by Young et al.

The Young et al. model has a black hole to core mass ratio $\mathscr{M}_{\bullet} / 4 \pi \rho_{0}\left(r_{\mathrm{c}} / 3\right)^{3}=0.6$; we plot this model in Fig. 7 as interpolated graphically from the models presented by Young (1980). The cusp model falls below our $\gamma=-0.26$ model by no more than $0.05 \mathrm{mag}$ for 0 " $1<r<1$ ". 0 , and begins to rise above it for $r<0$ ". 1 , just where the uncertainties become large. Young (1980) shows that the asymptotic power-law generated by the adiabatic growth of a black hole in an isothermal galaxy core with an isotropic velocity field will have $\mu \sim r^{-1 / 2}$ (or $\mu \sim r^{-3 / 4}$ if the stars are initially all on circular orbits); however, this form is approached only gradually as $r \rightarrow 0$, and over the regions well probed by the current observations, the apparent cusp slope will be much shallower, close to our average slope of $\gamma=-1 / 4$.

We plot luminosity density profiles in Fig. 8, deprojected with Abel transforms under the assumption of spherical symmetry, to show the true spatial distribution of stars in M87. Here the asymptotic slopes all decrease by $\Delta \gamma=-1$; the luminosity density profile in M87 is actually quite steep

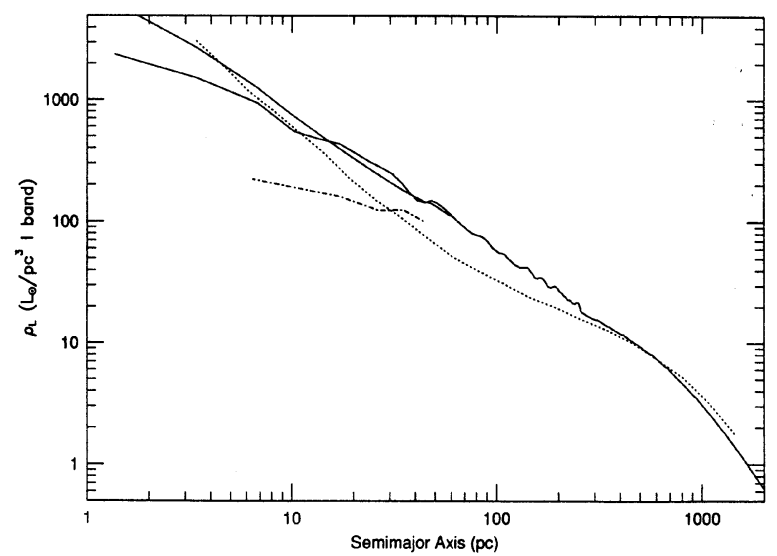

FIG. 8. Luminosity density profiles. The two solid lines show the deconvolved and $\gamma=-0.26$ power-law model starlight profiles deprojected as luminosity density profiles. The dotted line shows the Young et al. cusp model. The lower dashed segment shows the central behavior of the starlight density distribution assumed by Dressler \& Richstone (1990).

and has $\rho_{L}>10^{3} L_{\odot} \mathrm{pc}^{-3}$ ( $I$ band) for $r<10 \mathrm{pc}$. The Young (1980) model clearly shows the slope increasing as $r \rightarrow 0$; the disagreement between the observed profile and the Young model is larger in the density domain, but is still within the observational uncertainties.

The analysis presented here thus brings us right back to the original 1978 black hole model of Young et al.-but here lies a problem: while the light profile clearly suggests a massive black hole, the dispersion and rotation observations show little supporting evidence-and indeed appear to rule out the original Sargent et al. black hole, which had twice the mass of the Young et al. model based on the light profile. We suggest, however, that the smaller Young et al. black hole may in fact be the best explanation for all the data, and that it can probably be accommodated to the dynamics observations with only small adjustments of the models.

The dynamical observational picture of M87 is presented clearly by Dressler \& Richstone (1990). Their velocity dispersion profile flattens out to $\sigma=360 \pm 10 \mathrm{~km} / \mathrm{s}$ in the central 100 pc of M87; as argued earlier, we verify that their observations were properly centered. The Dressler \& Richstone (1990) dispersion profile is not evocative of the Sargent et al. dispersion cusp, and they argue that their data can rule out the $\mathscr{M}_{\bullet} \sim 5 \times 10^{9} \mathscr{M}_{\odot}$ Sargent et al. model directly. The game then is how well can one hide a massive black hole without it showing up in the existing dynamics observations. Dressler \& Richstone (1990) show that no black hole at all is required if they allow the central velocity ellipsoid to be somewhat radially biased. They can easily accommodate $\mathscr{M}_{\odot} \sim 1 \times 10^{9} \mathscr{M}_{\odot}$, by having the orbits close to the hole $(r<50 \mathrm{pc})$ be tangentially biased, but still radially biased outwards. As $\mathscr{M}_{\bullet}$ increases, the region of tangentially biased orbits increases, until $\mathscr{M}_{\bullet} \approx 3.6 \times 10^{9} \mathscr{M}_{\odot}$, at which all the central star orbits are nearly circular, and the projected dispersion begins to exceed the observational bounds.

Note that the range of permitted black holes includes the Young et al. $\mathscr{M}_{\bullet}=2.6 \times 10^{9} \mathscr{M}_{\odot}$. Moreover, if we take the present light profile and theoretical projected dispersion profile given by Young (1980), and "observe" the combination assuming the Dressler and Richstone seeing and slit 
parameters, we predict a measured central $\sigma \approx 385 \mathrm{~km} / \mathrm{s}$. It is a fair question whether this is already consistent with the central value of $\sigma=360 \pm 10 \mathrm{~km} / \mathrm{s}$ measured by Dressler and Richstone, given the systematic uncertainties associated with dispersion measurements; if not, then clearly only a modest reduction in hole mass will bring it in to line. Furthermore, the tangentially biased velocity ellipsoid required by Dressler and Richstone to accommodate the Young et al. black hole may in fact be physically reasonable. Both Young (1980) and Goodman \& Binney (1982) show that stellar orbits become significantly more tangentially biased as they are drawn into a growing black hole cusp. This is an intriguing effect that must now be carefully considered in future mass models of M87.

At this time, without further modeling, we cannot present conclusive arguments for $\mathscr{M}_{\bullet}>10^{9} \mathscr{M}_{\odot}$; however, we see several loose ends to be tied up that in themselves may lead to the answer. To start with, we note that the present light profile is significantly more concentrated than the model used by Dressler \& Richstone (see Fig. 8) and might in itself lead to a new kinematic picture regardless of any other refinements. It is also important to go beyond the assumptions inherent in the Young (1980) cusp models. In particular, models should be tried that do not start with an isotropic, isothermal core prior to introduction of the black hole. Rarely, if ever, could Lauer (1985a) or Kormendy (1985a) find such cores in other ellipticals that a priori might be considered more "normal" than M87. An HST survey is clearly needed to establish the baseline brightness profiles of normal cores.

On a deeper level, we need to consider the actual process whereby a black hole would form at the center of a galaxy. Young et al. assumed the adiabatic growth of a black hole in a preexisting core. The existence of QSOs at large lookback times, however, hints that massive black holes might have existed well before the complete galaxy formed around them (Turner 1991). Mergers or gaseous infall at late times could likewise modify the core well after a central black hole formed. In short, we need models that treat the joint formation of the core and hole self-consistently.

If there is not a black hole of significant mass at the center of M87, we still have the problem of explaining the light profile. Although we might be able to model the light distribution "microscopically" by clever selection of orbital families, we must answer why, physically, at $r \sim 700 \mathrm{pc}$ the light distribution makes a transition between a shallow power-law inside and a steep one outside. It is intriguing that this transition occurs at radii similar to the range of typical core radii for a galaxy of M87's luminosity (Lauer 1985a; Kormendy $1985 \mathrm{~b}$ ). In lieu of a black hole, this may suggest that M87 did once have a "normal" core and that the central power law we now see was created by late infall or a merger event. The question is then to what extent such a process could explain the light profile without leaving a distinctive kinematic signature.

In conclusion, we note that the present observations have now largely removed the ambiguity in the light profile that has stymied post-1978 analyses of M87. While Young et al. assumed a nonthermal spike, as recently as this year, Jarvis \& Melnick (1991) could offer a radically different model for the central light source. The uncertainty in the present profile is limited largely to $r<0$." 1 ; since the starlight cusp appears to start at $3^{\prime \prime}$, the present profile ought not to be a significant barrier to future interpretation. The outstanding problem now is to develop comprehensive models that incorporate realistic cores and follow the growth of the black hole in self-consistent fashion. It may soon be possible to obtain velocity dispersion measurements at $\sim 0$ ". 5 resolution from the ground, which will represent a factor of two improvement over the data now available. If the Young et al. $\mathscr{M}_{\bullet}=2.6 \times 10^{9} \mathscr{M}_{\odot}$ hole is really present, then one should see $\sigma \approx 460 \mathrm{~km} / \mathrm{s}$ in the central 0.5 square aperture in 0.5 FWHM seeing. Resolution finer than this may yet be required, however. This in our mind is excellent justification for restoring the full spatial resolution capabilities of the $H S T$ spectrographs.

This research was conducted by the WFPC Investigation Definition Team, supported in part by NASA Grant No. NAS5-1661.

\section{REFERENCES}

Bagnuolo, W. G., \& Chambers, R. W. 1987, Nature, 326, 681

Binney, J., \& Mamon, G. A. 1982, MNRAS, 200, 361

Biretta, J. A., Stern, C. P., \& Harris, D. E. 1991, AJ, 101, 1632

Bracewell, R. N. 1978, The Fourier Transform and its Applications (McGraw-Hill, New York)

Burrows, C., Holtzman, J. A., Faber, S. M., Bely, P., Husan, H., Lynds, C. R., \& Schroeder, D. 1991, ApJ, 369, L21

Dressler, A. 1980, ApJ, 240, L11

Dressler, A., \& Richstone, D. O. 1990, ApJ, 348, 120

Duncan, M. J., \& Wheeler, J. C. 1980, ApJ, 237, L27

Goodman, J., \& Binney, J. 1984, MNRAS, 207, 511

Griffiths, R. 1989, Wide Field and Planetary Camera Instrument Handbook (STScI, Baltimore, MD)

Högbom, J. A. 1974, A\&AS, 15, 417

Jarvis, B. J., \& Melnick, J. 1991, A\&A, 244, L1

Jarvis, B. J., \& Peltier, R. F. 1991, A\&A, 247, 315

Johnson, H. L. 1966, ARA\&A, 4, 193

Kéel, W. C. 1988, ApJ, 329, 532

Kormendy, J. 1985a, ApJ, 292, L9

Kormendy, J. 1985b, ApJ, 295, 73
Lauer, T. R. 1985a, ApJ, 292, 104

Lauer, T. R. 1985b, ApJS, 57, 473

Lauer, T. R. 1989, PASP, 101, 445

Lauer, T. R., \& Kormendy, J. 1986, ApJ, 303, L1

Lauer, T. R., et al. 1991, ApJ, 369, L41

Lucy, L. B. 1974, AJ, 79, 745

Perez-Fournon, I., Colina, L., Gonzalez-Serrano, J. I., \& Biermann, P. L. 1988, ApJ, 329, L81

Reid, M. J., Biretta, J. A., Junor, W., Muxlow, T. W. B., \& Spencer, R. E. 1989, ApJ, 336, 112

Richardson, W. H. 1972, J. Opt. Soc. Am., 62, 52

Richstone, D. O., \& Tremaine, S. 1985, ApJ, 296, 370

Sargent, W. L. W., Young, P. J., Boksenberg, A., Shortridge, K., Lynds, C. R., \& Hardwick, F. D. A. 1978, ApJ, 221, 731

Spencer, R. E., \& Junor, W. 1986, Nature, 321, 753

Turner, E. L. 1991, AJ, 101, 5

Young, P. J. 1980, ApJ, 242, 1232

Young, P. J., Westphal, J. A., Kristian, J., Wilson, C. P., \& Landauer, F. P. 1978, ApJ, 221, 721

Zavatti, F., Bendinelli, O., \& Parmeggiani, G. 1984, A\&A, 134, 99 


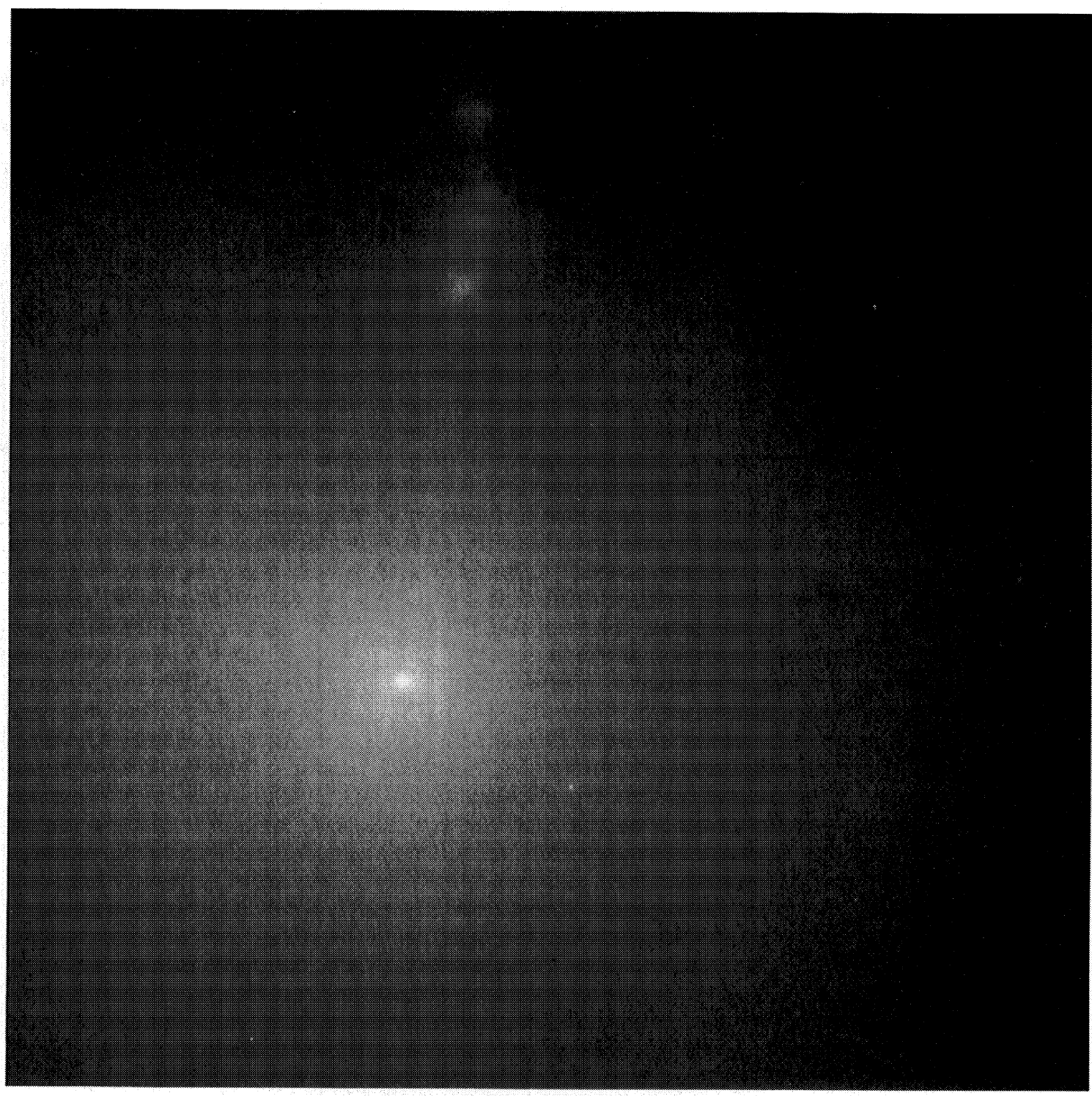

FIG. 1. PC image of M87 taken in filter F785LP. The area shown is the portion of the galaxy imaged by PC6 and is 765 pixels or 33 ".7 on a side. An arbitrary logarithmic stretch has been used. The center of the full Planetary Camera field is at the lower-left corner. The figure has normal celestial handedness. The vertical axis is rotated $60^{\circ}$ clockwise from north.

Lauer et al. (see page 705 ) 


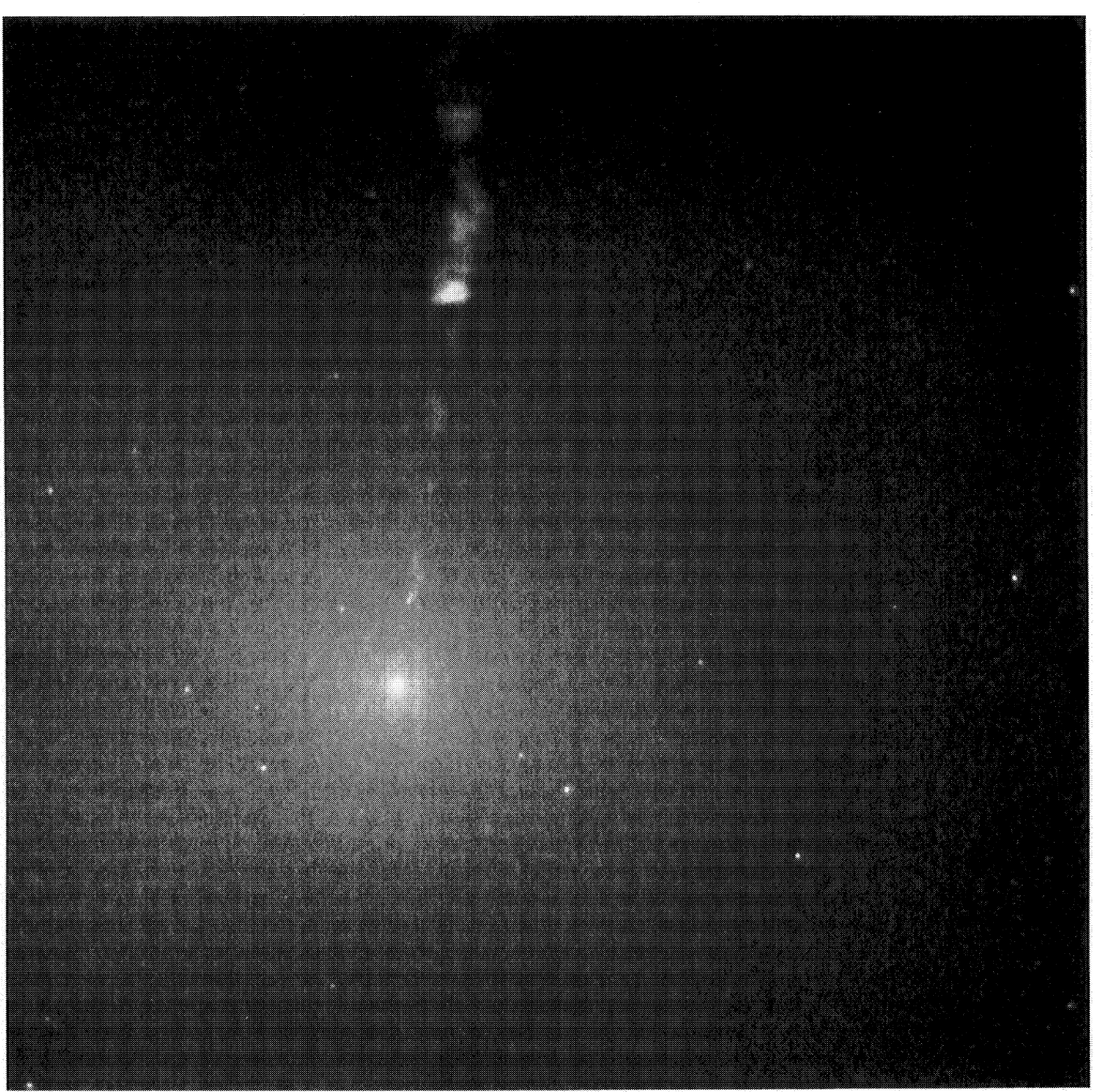

Fig. 2. Deconvolved image of M87. The image shown in Fig. 1 is shown again here after 80 Richardson-Lucy iterations. The stretch is logarithmic but has been set differently from Fig. 1 to show the compact nature of the central luminosity spike. The apparent elongation of the spike is due to the inner jet knots N1 and M. Also evident is the central gradient in the starlight distribution. The compact sources scattered about the image are globular clusters.

Lauer et al. (see page 705) 

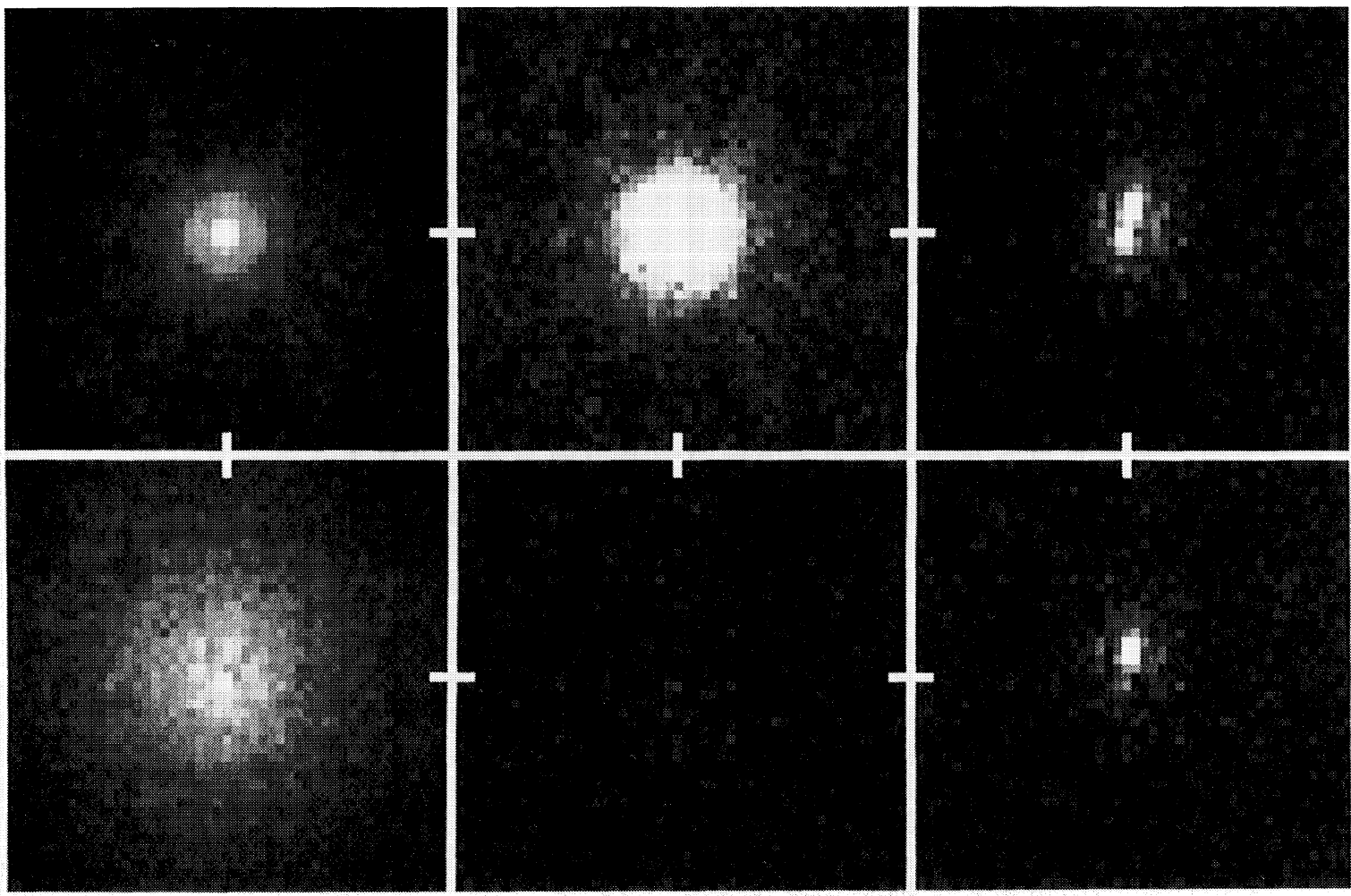

FIG. 4. Images of the inner starlight distribution, the central spike, and its decomposition. Each panel is 50 pixels or 2". 20 on a side. The tick marks point to the centroid of the luminosity spike. Orientation is as in Fig. 1. The dark feature seen in the upper left of all panels is a flatfield defect. The first panel in the upper left (a) is the central spike plus inner jet after subtraction of the power-law model with $\gamma=-0.26$ and $a=0$." 00 (see text). The stretch is logarithmic and set to show the stellar nature of the central spike. A central diffraction ring is evident, as is the strong PSF halo. The effect of the inner jet is subtly evident as a slight enhancement of the diffraction ring above the spike. The next panel to the right (b) is the same image but with a hard linear stretch. The upper-right panel (c) is shown with the same stretch, but with a pure spike subtracted. The two components left behind are the inner jet and a small source in the opposite direction that we associate with the spike (see text). The next panel in the lower right (d) shows the inner jet after subtraction of the residual spike component. The panel in the middle left (e) shows the residuals left after subtraction of the inner jet- this is what is left from the best fitting starlight model and the complete description of the nonthermal light. The largest residual is no more than $4 \%$ of the original flux. The final panel in the lower left ( $f$ ) is the central starlight left behind after subtraction of the nonthermal source models from the observations. The stretch is set so that black to white is a $0.5 \mathrm{mag}$ step in surface brightness; the central gradient is thus visible on subarcsecond scales.

Lauer et al. (see page 705) 